\title{
Dietary plant components ellagic acid and tannic acid inhibit Escherichia coli biofilm formation
}

Bacteria usually live as surface-associated communities, rather than as planktonic cells. These compact microbial consortia, referred to as biofilms, are commonly associated with many health problems (Costerton et al., 1999; Donlan, 2002). A few common examples of where biofilms form are in dental plaque, in lung infections and in infections related to the use of medical devices, such as catheters. Many persistent and chronic bacterial infections are now thought to be linked to biofilm formation; more than $60 \%$ of all bacterial infections have been estimated to involve biofilm formation (Lewis, 2001). Virtually all medical implants are prone to colonization and biofilm formation by pathogenic bacteria, and such biofilms can serve as a source for recurrent infections (Costerton et al., 1999). Biofilm-linked infections are particularly problematic, because biofilm-associated bacteria can tolerate immune defences, antibiotics, biocides and hydrodynamic shear forces far better than the corresponding planktonic bacteria. The intrinsic tolerance of biofilm-associated bacteria towards antimicrobial agents makes biofilmassociated infections particularly recalcitrant toward treatment. New types of antibiotics are therefore needed; development of candidate drugs that target biofilm formation will be of great importance. Most conventional antibiotics have targeted biochemical and physiological functions that are present both in pathogenic as well as saprophytic bacteria. Specific targeting of diseaseassociated bacterial lifestyles such as biofilm formation but not free-living pelagic bacteria is a highly attractive approach that has not yet been exploited. Drugs specifically aimed at bacterial biofilm formation are unlikely to be crossresistant to existing therapies.

Plants have been known for many years to contain health-improving substances. One example is green tea, which has been consumed for over 4000 years by a large number of people. Traditionally green tea has been recommended against infectious diseases and was frequently taken by patients suffering from infections (Hamilton-Miller, 1995). Dietary plant material contains numerous metabolites with antibacterial effects; the antibacterial properties have been attributed to the high level of polyphenols (30\% of dry weight in green tea) such as ellagic acid (EA) and tannic acid (TA) (Akiyama et al., 2001). On this background, we have investigated the anti-biofilm properties of these plant compounds.

TA and EA (Fig. 1a) were tested for their ability to inhibit biofilm formation by Escherichia coli. TA and EA were purchased from Sigma. Stock solutions of TA and EA (each $10 \mathrm{mg} \mathrm{ml}^{-1}$ ) were prepared in water and DMSO, respectively, and filter sterilized before use. MIC determinations were performed in FBA minimal medium (with $0.02 \%$ glucose, $0.02 \%$ Casamino acids, $1 \mu \mathrm{g}$ thiamine $\mathrm{ml}^{-1}$ and, in the case of VR50, $10 \mu \mathrm{g}$ panthotenic acid $\mathrm{ml}^{-1}$ ) according to the broth microdilution method and the concentrations used in the biofilm experiments were set to $<1 / 20$ of the MICs to ensure non-toxic conditions. Bacterial susceptibility was also assessed in urine, where the compounds showed no significant effect on planktonic growth under the conditions chosen for the biofilm experiments. The final concentration of TA and EA was $30 \mu \mathrm{g}$ $\mathrm{ml}^{-1}$. After addition of each of the tested compounds to the growth medium, the $\mathrm{pH}$ was checked and no change in $\mathrm{pH}$ due to the addition of compounds was observed.

Two E. coli strains with excellent biofilmforming abilities were chosen to monitor the biofilm-reducing potential of TA and EA, viz. VR50, a urinary tract infectious strain (Roos et al., 2006), and F18, a commensal isolate (Myhal et al., 1982). Both strains grew well in the presence of the two plant compounds and no reduction of growth was observed in the presence of the compounds at the concentrations used in the biofilm experiments (MIC values of $>600 \mu \mathrm{g}$ $\left.\mathrm{ml}^{-1}\right)$.

Biofilm formation in FBA minimal medium in 24-well flat-bottom microplates (Iwaki) at $37{ }^{\circ} \mathrm{C}$ of E. coli strains VR50 and F18 alone (control) or in the presence of EA and TA was monitored. TA and EA were added to static cultures at the time of inoculation. After incubation overnight, biofilm formation was determined by crystal violet staining as described previously (Ferrières et al., 2007) and the amount of biofilm was related to that of controls grown in medium without any additions. Each strain and compound was tested in three wells per plate and all experiments were repeated three to seven times. Both compounds reduced biofilm formation by VR50 and F18 significantly. TA and EA reduced biofilm formation by $44-80 \%$ and $22-26 \%$, respectively (Fig. 1b). In the presence of both TA and EA, no synergistic effect of the two compounds was observed (Fig. 1c).

To get rid of toxic substances and waste products, bacteria have efficient efflux systems. Efflux pumps occur both as single- and multi-component systems (Lee et al., 2000). Upon inhibition of these efflux pump systems, the intracellular concentration of toxic substances can reach critical levels, resulting in stress and/ or killing of cells (Marquez, 2005; Piddock, 2006). Efflux pump inhibitors (EPIs) have attracted a great deal of attention due to their ability to block some types of antibiotic resistance in bacteria (Marquez, 2005; Piddock, 2006); the EPIs known to block multidrug resistance pumps are of particular interest. Thioridazine (TZ; Fig. 1a) is a well-known EPI (Piddock, 2006). In a recent study, we demonstrated that $\mathrm{TZ}$ is an efficient inhibitor of biofilm formation in E. coli and Klebsiella pneumoniae (Kvist et al., 2008). These 
(a)

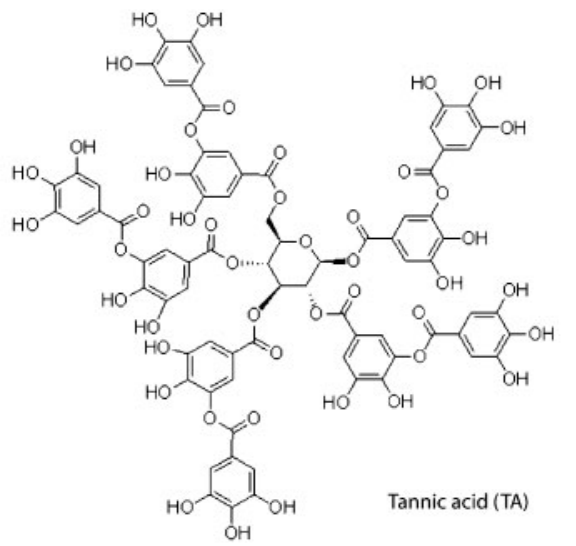<smiles>O=C1Oc2c(O)c(O)cc3c2C(=O)OC(O)=C1OC3=O</smiles>

Ellagic acid (EA)

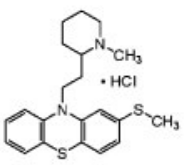

Thioridazine (TZ)

(b)

은

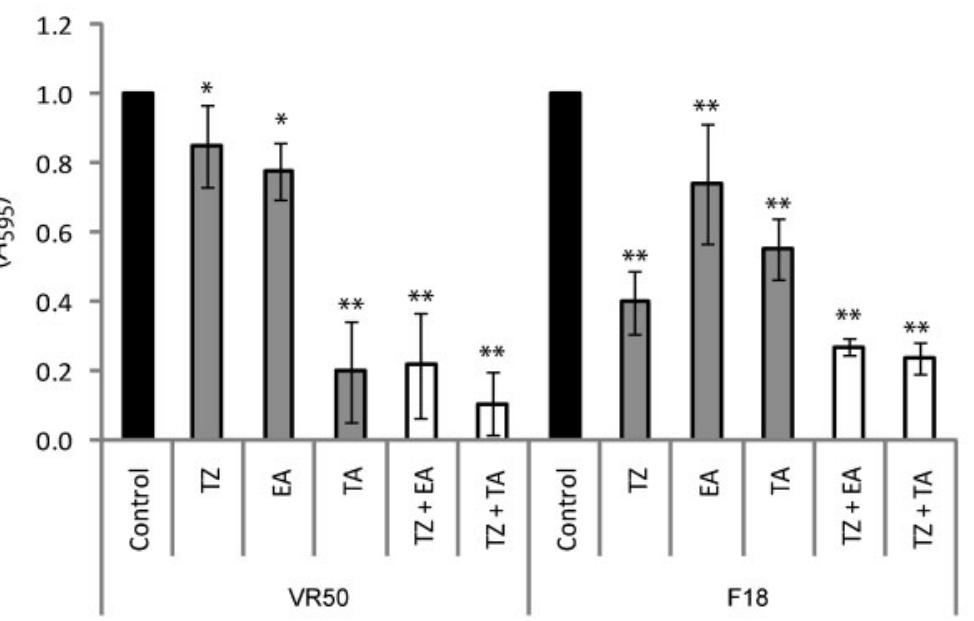

(c)

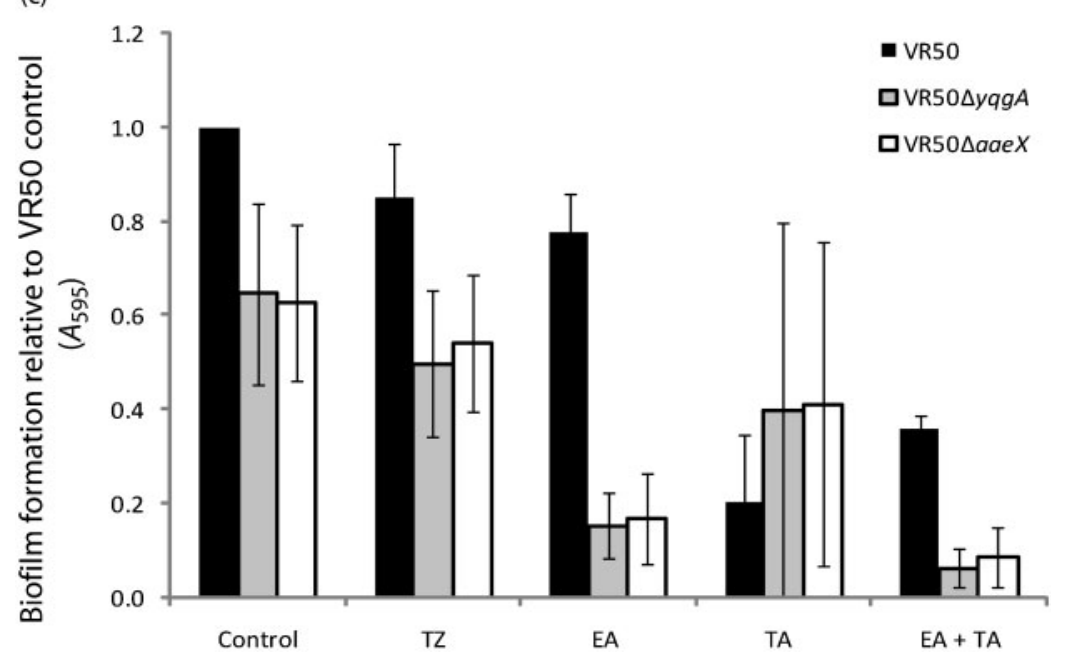

Fig. 1. (a) Molecular structures of the plant components TA and EA and of the EPI TZ used in the biofilm experiments. (b) Biofilm formation in FBA minimal medium of $E$. coli strains VR50 and F18 alone (control) or in the presence of TZ, EA and TA. Asterisks indicate significant reduction compared with the control (paired two-tailed $t$ test of at least three independent experiments; ${ }^{\star} P<0.05,{ }^{\star \star} P$ $<0.01)$. Bars indicate standard deviation. (c) Biofilm formation of $E$. coli UTI strain VR50 (black bars) and the two knockout mutants VR50 $4 y q g A$ (shaded bars) and VR50 $\Delta a a e X$ (white bars) in the presence of TZ, EA and TA. In all cases, except for TA, the two knockout mutants showed significant reduction in biofilm formation compared with VR50 grown with the same compound (paired two-tailed $t$ test of at least four independent experiments; $P<0.01$ ). Bars indicate standard deviation. results suggest the use of EPIs as novel anti-biofilm agents. Biofilm formation in the presence of $\mathrm{TZ}$ was also monitored. TZ was purchased from Sigma and a stock solution $\left(10 \mathrm{mg} \mathrm{ml}^{-1}\right)$ was prepared in water and filter sterilized. The MIC was determined as described for EA and TA, and the concentration used in the biofilm experiments $\left(40 \mu \mathrm{g} \mathrm{ml}^{-1}\right)$ was set to $1 / 5$ of the MIC. TZ reduced biofilm formation in
VR50 and F18 by 20\% and 60\%, respectively (Fig. 1b), at 1/5 MIC; MIC values for both strains were $200 \mu \mathrm{g} \mathrm{ml}^{-1}$. With this in mind, we wanted to test the effect of TA and EA in combination with 
TZ. In the case of strain VR50, the biofilmreducing effect of EA was significantly enhanced in the presence of TZ, i.e. from $22 \%$ to $78 \%$ (Fig. 1b). For both E. coli strains tested, the combination of $\mathrm{TZ}$ with either TA or EA reduced biofilm formation by between $73 \%$ and $89 \%$ (Fig. 1b).

Two knockout mutants of VR50 with deletions in transport/efflux systems, i.e.

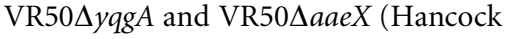
et al., 2007), showed significant reduction in biofilm formation in FBA minimal medium (35-37\%). These mutants have previously been shown to have decreased biofilm formation (Hancock et al., 2007). In the presence of EA, the mutants showed a significantly greater reduction in biofilm formation, 78-80\%, compared with the wild-type (Fig. 1c). In the presence of TA, however, the mutants showed no significant difference compared with wildtype. Despite the fact that no synergistic effect of the two plant acids on biofilm formation by the wild-type could be observed, the very different effects of the plant compounds on the knockout mutants suggest that they act by different mechanisms on biofilm formation (Fig. 1c).

The mechanism responsible for the antibacterial effects of tea and other plant compounds is somewhat elusive; plant secondary metabolites, to a large extent polyphenols, show bactericidal effects as a result of damage to the cell membrane (Maeyama et al., 2005). However, we observed no growth reduction of bacteria growing in liquid cultures with the concentrations used for the biofilm experiments. Therefore, the mechanism by which EPIs potentiate the use of antibiotics (vide supra) does not necessarily apply to the effect observed in the case where the EPI TZ was used in combination with our plant compounds.
In conclusion, we have presented results indicating that secondary metabolites, such as TA and EA, from plants act as antibiofilm agents. Such substances are worthy of attention in the fight against bacterial infections. Contrary to classical antibiotics that generally kill bacteria, the plant compounds reported on here rather seem to modify the behaviour of bacteria, i.e. they abolish biofilm formation but have no or little effect on planktonic bacteria. In effect, they can be considered as lifestylemodifying drugs. Hopefully, such compounds might not be as prone as classic antibiotics to the development of bacterial resistance.

\section{Acknowledgements}

We thank Birthe Jul Jørgensen for expert technical assistance. This work was supported by grants from the Danish Medical Research Council (Grant 271-07-0291), Lundbeckfonden (R19-A2191) and FØSU (Grant 2101-06-0009).

\section{Viktoria Hancock, Malin Dahl, Rebecca Munk Vejborg and Per Klemm}

Microbial Genomics Group, Center for

Biological Sequence Analysis,

Department of Systems Biology,

Technical University of Denmark, Lyngby, Denmark

\section{Correspondence: Per Klemm (pkl@cbs.dtu.dk)}

Akiyama, H., Fujii, K., Yamasaki, O., Oono, T. \& Iwatsuki, K. (2001). Antibacterial action of several tannins against Staphylococcus aureus. J Antimicrob Chemother 48, 487-491.

Costerton, J. W., Stewart, P. S. \& Greenberg, E. P. (1999). Bacterial biofilms: a common cause of persistent infections. Science 284, $1318-1322$.
Donlan, R. M. (2002). Biofilms: microbial life on surfaces. Emerg Infect Dis 8, 881-890.

Ferrières, L., Hancock, V. \& Klemm, P. (2007). Biofilm exclusion of uropathogenic bacteria by selected asymptomatic bacteriuria Escherichia coli strains. Microbiology 153, 1711-1719.

Hamilton-Miller, J. M. T. (1995). Antimicrobial properties of tea (Camellia sinensis L.). Antimicrob Agents Chemother 39, 23752377.

Hancock, V., Ferrières, L. \& Klemm, P. (2007). Biofilm formation by asymptomatic and virulent urinary tract infectious Escherichia coli strains. FEMS Microbiol Lett 267, 30-37.

Kvist, M., Hancock, V. \& Klemm, P. (2008). Inactivation of efflux pumps abolishes bacterial biofilm formation. Appl Environ Microbiol 74, 7376-7382.

Lee, A., Mao, W., Warren, M. S., Hoshino, K., Okumura, R., Ishida, H. \& Lomovskaya, O.

(2000). Interplay between efflux pumps may provide either additive or multiplicative effects on drug resistance. J Bacteriol 182, 31423150.

Lewis, K. (2001). Riddle of biofilm resistance. Antimicrob Agents Chemother 45, 999-1007.

Maeyama, R., Kwon, I. K., Mizunoe, Y., Anderson, J. M., Tanaka, M. \& Matsuda, T. (2005). Novel bactericidal surface: catechinloaded surface-erodible polymer prevents biofilm formation. J Biomed Mater Res A 75, 146-155.

Marquez, B. (2005). Bacterial efflux systems and efflux pumps inhibitors. Biochimie 87, 11371147.

Myhal, M. L., Laux, D. C. \& Cohen, P. S. (1982). Relative colonizing abilities of human fecal and K-12 strains of Escherichia coli in the large intestines of streptomycin-treated mice. Eur J Clin Microbiol 1, 186-192.

Piddock, L. J. V. (2006). Clinically relevant chromosomally encoded multidrug resistance efflux pumps in bacteria. Clin Microbiol Rev 19, 382-402.

Roos, V., Nielsen, E. M. \& Klemm, P. (2006). Asymptomatic bacteriuria Escherichia coli strains: adhesins, growth and competition. FEMS Microbiol Lett 262, 22-30. 